\title{
Phonon Contribution in Thermodynamics of Nano-Crystalline Films and Wires
}

\author{
J.P. Šetrajčića ${ }^{a, f, *}$, D.Lu. Mirjanić $b$, S.M. VuČenović $b$, D.I. Ilićc ${ }^{c}$, B. Markoski ${ }^{c}$ \\ S.K. JAĆIMOVSKI ${ }^{d}$, V.D. SAJfERT ${ }^{e}$ AND V.M. ZORIĆ ${ }^{a}$ \\ ${ }^{a}$ Department of Physics, Faculty of Sciences, University of Novi Sad \\ Trg D. Obradovića 4, 21000 Novi Sad, Vojvodina, Serbia \\ ${ }^{b}$ Faculty of Medicine, University of Banja Luka, Republic of Srpska, BIH \\ ${ }^{c}$ Faculty of Technical Sciences, University of Novi Sad, Vojvodina, Serbia \\ ${ }^{d}$ Police Academy, Belgrade, Serbia \\ ${ }^{e}$ Technical Faculty "M. Pupin", University of Novi Sad, Vojvodina, Serbia \\ ${ }^{f}$ Academy of Sciences and Arts, Banja Luka, Republic of Srpska, BIH
}

\begin{abstract}
Spectra of possible phonon states, as well as thermodynamic characteristics of nanocrystals (ultrathin film and quantum wire) of simple cubic crystalline structure are analyzed in this paper, using the method of two-time dependent Green functions. From energy spectra and internal energy of the system the thermal capacitance of these structures in low temperature region is found. The temperature behavior of specific heat is compared to that of corresponding bulk structure. It is shown that at extremely low temperatures thermal capacitance of quantum wire is considerably lower than the thermal capacitance of film as well as the bulk sample. Consequences of this fact are discussed in detail and its influence to thermodynamic properties of materials is estimated.
\end{abstract}

PACS numbers: 63.20.-e, 65.40.Ba, 67.25.dp, 68.60.-p, 68.65.La

\section{Introduction}

Crystalline films are confined crystalline structures [1-3] in which translational symmetry is broken along direction perpendicular to the film (z-direction, Fig. 1a). Calculation of thermodynamic characteristics of phonons in film structures was presented in a previous YUCOMAT conference [4]. Now we have calculated phonon contribution in thermodynamic characteristics of quantum wires and compared these results with previous ones in ultrathin film structures.

Quantum wires are confined crystalline structures too, but in which conditions on boundary surfaces differ from those inside the wires, i.e. translational symmetry is broken along directions perpendicular to the wire $(z$ and $y$ directions, Fig. 1b). Providing that there is no disturbance of the crystalline structure inside the wire (between its boundary surfaces), we assume that quantum wire is ideal. On the contrary, if there are impurities, vacancies and the like in the crystalline lattice, quantum wire is deformed. The scope of our study in this paper is the ideal quantum wire of simple cubic crystalline structure consisting of identical atoms, made on the substrate with

\footnotetext{
* corresponding author; e-mail:

bora@if.ns.ac.yu,vojzoric@yahoo.com
}

the use of some appropriate technological process (deposition, sputtering, etc.), whose basic crystallographic parameters - in the nearest neighbors approximation are [5-7]:

$$
\begin{aligned}
& a_{x}=a_{y}=a_{z} \equiv a, \quad N_{x} \sim 10^{8} \gg N_{y, z} \sim 10, \\
& C_{\boldsymbol{n}, \boldsymbol{m}}^{\alpha, \alpha}=C_{\boldsymbol{n}, \boldsymbol{n} \pm \boldsymbol{\lambda}}^{\alpha} \equiv C_{n_{x}, n_{y}, n_{z} ; n_{x} \pm 1, n_{y}, n_{z}}^{\alpha}= \\
& \quad C_{n_{x}, n_{y}, n_{z} ; n_{x}, n_{y} \pm 1, n_{z}}^{\alpha}= \\
& \quad C_{n_{x}, n_{y}, n_{z} ; n_{x}, n_{y}, n_{z} \pm 1}^{\alpha} \equiv C^{\alpha}, \\
& C_{n_{y}, 0 ; n_{y},-1}^{\alpha}=C_{n_{y},-1 ; n_{y}, 0}^{\alpha}=(1+\varepsilon) C^{\alpha}, \\
& C_{n_{y}, N_{z} ; n_{y}, N_{z}+1}^{\alpha}=C_{n_{y}, N_{z}+1 ; n_{y}, N_{z}}^{\alpha}=(1+\gamma) C^{\alpha}, \\
& C_{0, n_{z} ;-1, n_{z}}^{\alpha}=C_{-1, n_{z} ; 0, n_{z}}^{\alpha}=(1+\sigma) C^{\alpha}, \\
& C_{N_{y}, n_{z} ; N_{y}+1, n_{z}}^{\alpha}=C_{N_{y}+1, n_{z} ; N_{y}, n_{z}}^{\alpha}=(1+\phi) C^{\alpha}, \\
& \quad \varepsilon, \gamma, \sigma, \phi \geq-1,
\end{aligned}
$$

where $a$ is the lattice constant, $N_{x, y, z}$ are the numbers of atoms along $x, y$ and $z$ directions, $C^{\alpha}$ is the strain Hooke elastic constant in direction $\alpha, n_{y, z} \in\left(0,1,2, \ldots, N_{y, z}\right)$ is the atom site counter along $y$ and $z$ directions; vector $\boldsymbol{\lambda}$ associates atom in place $\boldsymbol{n}$ with its nearest neighbors.

Respecting above facts, we are able to say the following about the described model structure: 

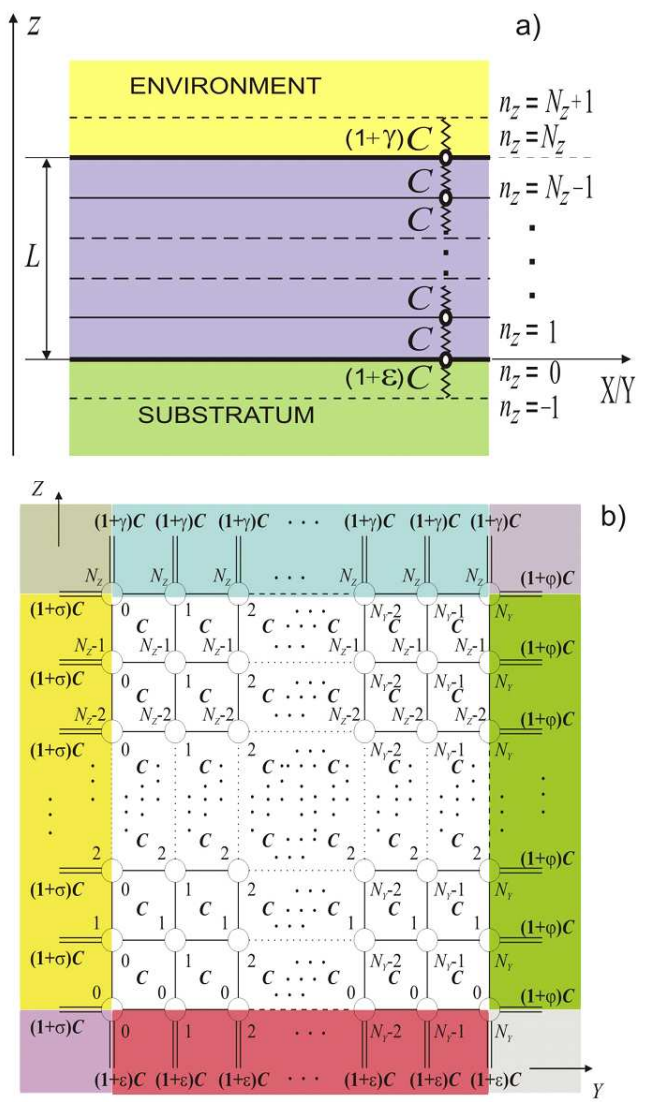

Fig. 1. Cross-section of the model of ultrathin film (a) and quantum wire (b).

1. Quantum wires have four boundary surfaces: two of them are parallel to the $X Y$ planes (for $z=0$ and $z=L_{z}=N_{z} a$ ), and two are parallel to $X Z$ planes (for $y=0$ and $y=L_{y}=N_{y} a$ ), thus, these structures are confined along $y$ and $z$ directions, while unbound along $x$ direction. Along $y$-axis there are $N_{y}+1$ atoms, and along $z$-axis $N_{z}+1$ atoms.

2. Torsion Hooke's elastic constants $C^{\alpha \beta}$ are negligible relative to the strain constants $C^{\alpha}$. It is considered that there is an interaction between atoms in boundary layers of the quantum wire and external areas, disregarding that along $y$ and $z$ directions there are no atoms of quantum wire; however, boundary atoms are coupled through changed Hooke's forces with the atoms of external environment. In accordance with these conditions, elastic constants which describe interaction between atoms of boundary surfaces and external environment are modified with appropriate coefficients $\varepsilon$ and $\gamma, \sigma$ and $\phi$. These perturbations of boundary surfaces do not disturb the macroscopic geometry of the structure, but only redefine small atom movements.

With respect to described model and regarding the fact that layers with $n_{y} \leq-1$ and $n_{y} \geq N_{y}+1$, and also $n_{z} \leq-1$ and $n_{z} \geq N_{z}+1$ are not present, we have to take into account the following:

$$
\begin{aligned}
& u_{\alpha ; n_{x} n_{y} n_{z}}=0 \quad-1 \geq n_{y, z} \wedge n_{y, z} \geq N_{y, z}+1, \\
& n_{y, z} \notin\left[0, N_{y, z}\right] .
\end{aligned}
$$

\section{Theoretical analysis}

Starting point of our theoretical approach is the standard Hamiltonian of the phonon subsystem for bulk structures [5-8], written in the harmonic as well as in the nearest neighbor approximations, which is adapted to the model-structure of quantum wire presented in Fig. 1b:

$$
\begin{aligned}
H & =T+V_{\mathrm{eff}}, \quad T=\sum_{\alpha ; \boldsymbol{n}} \frac{p_{\alpha ; \boldsymbol{n}}^{2}}{2 M} \\
V_{\mathrm{eff}} & =\sum_{\alpha ; n_{x}, n_{y}, n_{z}} \frac{C^{\alpha}}{4}\left[\left(u_{\alpha ; n_{x}+1, n_{y}, n_{z}}-u_{\alpha ; n_{x}, n_{y}, n_{z}}\right)^{2}\right. \\
& +\left(u_{\alpha ; n_{x}-1, n_{y}, n_{z}}-u_{\alpha ; n_{x}, n_{y}, n_{z}}\right)^{2} \\
& +\left(u_{\alpha ; n_{x}, n_{y}+1, n_{z}}-u_{\alpha ; n_{x}, n_{y}, n_{z}}\right)^{2} \\
& +\left(u_{\alpha ; n_{x}, n_{y}-1, n_{z}}-u_{\alpha ; n_{x}, n_{y}, n_{z}}\right)^{2} \\
& +\left(u_{\alpha ; n_{x}, n_{y}, n_{z}+1}-u_{\alpha ; n_{x}, n_{y}, n_{z}}\right)^{2} \\
& \left.+\left(u_{\alpha ; n_{x}, n_{y}, n_{z}-1}-u_{\alpha ; n_{x}, n_{y}, n_{z}}\right)^{2}\right],
\end{aligned}
$$

where $u_{\alpha ; \boldsymbol{n}}$ are the small movements of atom in place $\boldsymbol{n}$ from its equilibrium position in direction $\alpha$, and $p_{\alpha ; \boldsymbol{n}}$ corresponding momentum. $M$ is the mass of the atoms.

We are looking for the phonon dispersion law with the help of the phonon two-time commutator Green function $[8,9]$ :

$$
\begin{gathered}
G_{\boldsymbol{n}, \boldsymbol{m}}^{\alpha}\left(t-t^{\prime}\right) \equiv\left\langle\left\langle u_{\alpha ; \boldsymbol{n}}(t) \mid u_{\alpha ; \boldsymbol{m}}\left(t^{\prime}\right)\right\rangle\right\rangle= \\
\Theta\left(t-t^{\prime}\right)\left\langle\left[u_{\alpha ; \boldsymbol{n}}(t), u_{\alpha ; \boldsymbol{m}}\left(t^{\prime}\right)\right]\right\rangle_{0},
\end{gathered}
$$

which satisfies the equation of motion

$$
\begin{gathered}
-M \omega^{2} G_{\boldsymbol{n}, \boldsymbol{m}}^{\alpha}(\omega)=-\frac{\mathrm{i} \hbar}{2 \pi} \delta_{\boldsymbol{n}, \boldsymbol{m}} \\
+\frac{1}{\mathrm{i} \hbar}\left\langle\left\langle\left[p_{\alpha ; \boldsymbol{n}}, H\right] \mid u_{\alpha ; \boldsymbol{m}}\right\rangle\right\rangle_{\omega} .
\end{gathered}
$$

By calculating corresponding commutators in the Green function which appears in Eq. (1), and by using the partial spatial Fourier transformation (because the translational symmetry has been disturbed along $y$ and $z$ directions):

$$
\begin{aligned}
& G_{n_{x}, n_{y}, n_{z} ; \boldsymbol{m}}^{\alpha}(\omega)= \\
& \quad \frac{1}{N_{x}} \sum_{k_{x}} \mathrm{e}^{\mathrm{i} k_{x} a\left(n_{x}-m_{x}\right)} G_{n_{y}, n_{z} ; m_{y}, m_{z}}^{\alpha}\left(k_{x} ; \omega\right), \\
& \delta_{n_{x}, m_{x}} \delta_{n_{y}, m_{y}} \delta_{n_{z}, m_{z}}= \\
& \quad \frac{1}{N_{x}} \sum_{k_{x}} \mathrm{e}^{\mathrm{i} k_{x} a\left(n_{x}-m_{x}\right)} \delta_{n_{y}, m_{y}} \delta_{n_{z}, m_{z}},
\end{aligned}
$$

we obtain the system of $\left(N_{y}+1\right) \times\left(N_{z}+1\right)$ non- 
-homogeneous algebraic-difference equations with the same number of undetermined Green's functions [5-7]:

where

$$
\begin{aligned}
& G_{n_{y}-1, n_{z}}^{\alpha}+G_{n_{y}, n_{z}}^{\alpha}+\rho_{\boldsymbol{k}}^{\alpha} G_{n_{y}, n_{z}}^{\alpha}+G_{n_{y}, n_{z}+1}^{\alpha} \\
& \quad+G_{n_{y}+1, n_{z}}^{\alpha}=K_{n_{y}, n_{z}}
\end{aligned}
$$

$$
\begin{aligned}
& G_{n_{y}, n_{z}}^{\alpha} \equiv G_{n_{y}, n_{z} ; m_{y}, m_{z}}^{\alpha}\left(k_{x} ; \omega\right), \\
& K_{n_{y}, n_{z}}=\frac{\mathrm{i} \hbar}{2 \pi C_{\alpha}} \delta_{n_{y}, n_{z} ; m_{y}, m_{z}}, \\
& \rho_{\boldsymbol{k}}^{\alpha}=\frac{\omega^{2}}{\Omega_{\alpha}^{2}}-4\left(1+\sin ^{2} \frac{a k_{x}}{2}\right), \quad \Omega_{\alpha}^{2}=C_{\alpha} / M .
\end{aligned}
$$

In order to find the spectra of the allowed phonon energies amounts, we must determine the roots of the determinant of the system of Eqs. (2). This task in general is not analytically solvable (it can be solved numerically with given parameters $\varepsilon, \gamma, \sigma, \phi, N_{y}$ and $N_{z}$ ). Hereafter, we will give our attention to the model of the loose surfaces $[1,2]$, when surface perturbations are negligible, i.e. $\varepsilon=\phi=\gamma=\sigma=0$. Undetermined Green's functions from Eq. (2) can be expressed as follows:

$$
G_{n_{y}, n_{z}}=\frac{\mathcal{D}_{n_{y}, n_{z}}}{\mathcal{D}_{N_{y}+1, N_{z}+1}},
$$

where $\mathcal{D}_{n_{y}, n_{z}}$ is the determinant of the variable and $\mathcal{D}_{N_{y}+1, N_{z}+1}$ the two-dimensional determinant of the system. Poles of Green's functions by which the phonon dispersion law is determined can be obtained on condition that the determinant of the system is equal to zero: $\mathcal{D}_{N_{y}+1, N_{z}+1} \equiv 0$.

Determinant $\mathcal{D}_{N_{y}+1, N_{z}+1}$ can be expressed through Chebyshev's polynomials of second order, by which the phonon dispersion law can be obtained in the form

$$
\begin{gathered}
\varepsilon_{\boldsymbol{k}}^{\alpha}=2 \sqrt{\sin ^{2} \frac{a k_{x}}{2}+\sin ^{2} \frac{a k_{y}(\mu)}{2}+\sin ^{2} \frac{a k_{z}(\nu)}{2},} \\
\mu=1,2, \ldots N_{y}+1, \quad \nu=1,2, \ldots N_{z}+1,
\end{gathered}
$$

similar to that of the bulk structures. Main difference is, however, that phonon quasi-momentum in quantum wires takes discrete values in $y$ and $z$ directions and is continual in $x$ direction. It can also be seen that minimum phonon energy in quantum wires differs from zero, and is given by

$$
\begin{aligned}
& \Delta_{\min }=\varepsilon_{\boldsymbol{k}_{\text {min }}}^{\alpha}=\varepsilon_{k_{x}=0, k_{y, z}=k_{y, z}^{\min }}^{\alpha}= \\
& 2 \sqrt{\sin ^{2} \frac{a k_{y}^{\min }(\mu)}{2}+\sin ^{2} \frac{a k_{z}^{\min }(\nu)}{2}}, \\
& k_{y}^{\min }(\mu)=k_{y}(1)=\frac{\pi}{a} \frac{1}{N_{y}+2}, \\
& k_{z}^{\min }(\nu)=k_{z}(1)=\frac{\pi}{a} \frac{1}{N_{z}+2},
\end{aligned}
$$

while the corresponding minimum phonon frequency is

$$
\omega_{\min }^{w}=2 \frac{v}{a} \sqrt{\sin ^{2} \frac{\pi}{2\left(N_{y}+2\right)}+\sin ^{2} \frac{\pi}{2\left(N_{z}+2\right)}} \neq 0 .
$$

In Fig. 2 there are graphically presented phonon spectra in ultrathin film (a): $\varepsilon=\varepsilon_{\nu}\left(X^{2}+Y^{2}\right)$, where $X \equiv \sin \left(a k_{x} / 2\right)$ and $Y \equiv \sin \left(a k_{y} / 2\right)$, for $\nu=4$ (from [6]) and quantum wires (b): $\varepsilon=\varepsilon_{\mu, \nu}\left(X^{2}\right)$, where $X \equiv \sin \left(a k_{x} / 2\right)$, for $\mu=5$ and $\nu=4$. Broken lines represent continual zone of allowed phonon energy in bulk structures. Energy gaps and energy discreteness, which are sole consequences of existence of spatial boundaries, are observable.
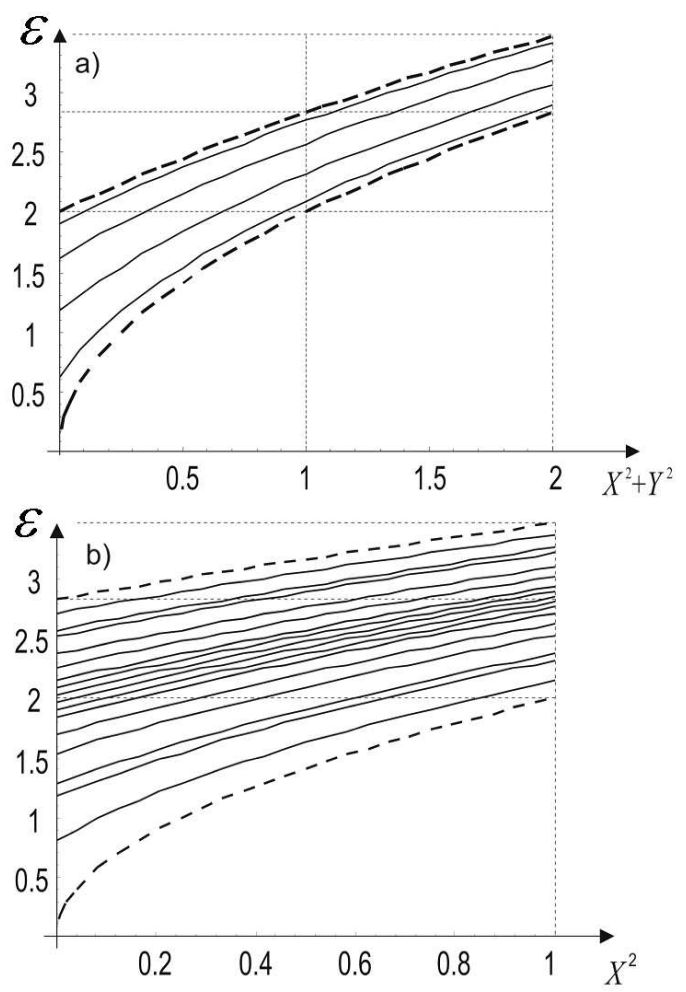

Fig. 2. Phonon spectra in ultrathin films (a) and in quantum wires (b).

\section{Thermodynamics of quantum wires}

In order to determine thermodynamic properties of quantum wires it is necessary to find corresponding values for the Debye wave vector and the Debye frequency. We assume that phonon wave vectors of quantum wire lie in sphere of radius $k_{\mathrm{D}}^{w}$. Since translational symmetry of quantum wire is broken along $y$ and $z$ directions, possible values of wave vector along $x, y$, and $z$ directions are

$$
\begin{aligned}
& k_{x} \in\left[-\frac{\pi}{a},+\frac{\pi}{a}\right] \Rightarrow \Delta k_{x}=\frac{2 \pi}{a}, \\
& k_{y / z} \in\left[\frac{1}{N_{y / z}+2} \frac{\pi}{a}, \frac{N_{y / z}+1}{N_{y / z}+2} \frac{\pi}{a}\right] \Rightarrow \Delta k_{y / z}= \\
& \frac{N_{y / z}}{N_{y / z}+2} \frac{\pi}{a}
\end{aligned}
$$

and we obtain 


$$
\begin{gathered}
V=\left\{\begin{array}{l}
4 \pi\left(a k_{\mathrm{D}}^{w}\right)^{3} / 3 \\
a^{3} \Delta k_{x} \Delta k_{y} \Delta k_{z}
\end{array} \Rightarrow k_{\mathrm{D}}^{w}=\right. \\
\sqrt[3]{\frac{3 \pi^{2}}{2 a^{3}} \sqrt[3]{\frac{N_{y}}{N_{y}+2}} \sqrt[3]{\frac{N_{z}}{N_{z}+2}}=} \\
k_{\mathrm{D}}^{b} \sqrt[3]{\frac{N_{y}}{2\left(N_{y}+2\right)} \frac{N_{z}}{2\left(N_{z}+2\right)}},
\end{gathered}
$$

where $k_{\mathrm{D}}^{b}=\sqrt[3]{6 \pi^{2}} / a$ is the Debye wave vector in corresponding unbound (bulk) structure. For the number of allowed values of $\boldsymbol{k}$ per volume unit of $\boldsymbol{k}$-space, next adjusted expression is applicable

$$
\begin{gathered}
D_{w}(\omega)=\frac{3 N_{x} N_{z}^{w} N_{z}^{w} a^{3}}{(2 \pi)^{3}} \int_{0}^{\pi} \sin \vartheta \mathrm{d} \vartheta \int_{0}^{2 \pi} \mathrm{d} \phi \\
\quad \times \int_{0}^{k_{\mathrm{D}}} k^{2} \mathrm{~d} k \delta(\omega-v k)=\frac{N_{x} N_{z}^{w} N_{z}^{w} a^{3}}{2 \pi^{2}} \frac{\omega^{2}}{v^{3}},
\end{gathered}
$$

and by applying the normalization condition (according to fact that the total number of phonon states is equal to the number of atoms):

$$
\begin{gathered}
\int_{0}^{\omega_{\mathrm{D}}^{w}} D_{w}(\omega) \mathrm{d} \omega=N \Rightarrow \frac{N_{x} N_{z}^{w} N_{z}^{w} a^{3}}{2 \pi^{2}} \frac{\omega^{2}}{v^{3}} \\
\times \int_{0}^{\omega_{\mathrm{D}}^{w}} \omega^{2} \mathrm{~d} \omega=N_{x}\left(N_{y}^{w}+1\right)\left(N_{z}^{w}+1\right),
\end{gathered}
$$

we obtain an expression for the Debye frequency in quantum wire in the form

$$
\omega_{\mathrm{D}}^{w}=\omega_{\mathrm{D}}^{b} \sqrt[3]{\frac{N_{y}^{w}+1}{N_{y}^{w}} \frac{N_{z}^{w}+1}{N_{z}^{w}}},
$$

where $\omega_{\mathrm{D}}^{b}=k_{\mathrm{D}}^{b} v$ is the Debye frequency in corresponding bulk structure. It can be seen that the Debye frequency has somewhat greater value in quantum wire than in unbound structure.

Internal energy of quantum wire is calculated in terms of standard form

$$
\begin{gathered}
U_{w}=\int_{0}^{\omega_{\mathrm{D}}^{w}} \mathrm{~d} \omega_{w} D_{w}(\omega)\left\langle n\left(\omega_{w}, T\right)\right\rangle \hbar \omega_{w}= \\
\int_{0}^{\omega_{\mathrm{D}}^{w}} \mathrm{~d} \omega_{w} \frac{\omega_{w}^{2} V}{2 \pi^{2} v^{3}}\left(\frac{\hbar \omega_{w}}{\mathrm{e}^{\frac{\hbar \omega_{\mathrm{B}}}{k_{\mathrm{B}}}}-1}\right)= \\
9 N_{w} k_{\mathrm{B}} T\left(\frac{T}{T_{\mathrm{D}}^{b}}\right)^{3} \int_{0}^{x_{\mathrm{D}}^{w}} \mathrm{~d} x_{w} \frac{\left(x_{w}\right)^{3}}{\mathrm{e}^{x_{w}}-1},
\end{gathered}
$$

where $V=N_{w} a^{3}, N_{w} \equiv N_{x}\left(N_{y}^{w}+1\right)\left(N_{z}^{w}+1\right)$, $x_{w}=\frac{\hbar \omega_{w}}{k_{\mathrm{B}} T}$ and $x_{\mathrm{D}}^{w}=\frac{\hbar \omega_{\mathrm{D}}^{w}}{k_{\mathrm{B}} T}=\frac{T_{\mathrm{D}}^{w}}{T}$.

In low temperature region, when $x_{\mathrm{D}}^{w} \rightarrow \infty$, the last expression becomes

$$
\begin{aligned}
& U_{w}=9 N_{w} k_{\mathrm{B}} T\left(\frac{T}{T_{\mathrm{D}}^{b}}\right)^{3} \int_{0}^{\infty} \mathrm{d} x_{w} \frac{\left(x_{w}\right)^{3}}{\mathrm{e}^{x_{w}}-1}= \\
& \frac{3 \pi^{4}}{5} N_{w} k_{\mathrm{B}} T\left(\frac{T}{T_{\mathrm{D}}^{b}}\right)^{3} .
\end{aligned}
$$

To find the expression for the thermal capacitance per unit cell (here: per atom), the standard definitional form is used: $C_{w}^{*}=\frac{1}{N_{w}} \frac{\partial U_{w}}{\partial T}$. In accordance with that we obtain

$$
C_{w}^{*}=\frac{12}{5} \pi^{4} k_{\mathrm{B}} \tau_{w}^{3}
$$

where $\tau_{w}=T / T_{\mathrm{D}}^{b}$. It is well known that the phonon part in thermal capacitance of the system is described with cubic temperature dependence. For comparison of these dependences for bulk structure and quantum wire, the last expression is divided by the constant $C_{0}=12 \pi^{4} k_{\mathrm{B}} / 5$, whose dimension is equal to dimension of thermal capacitance $C \equiv \frac{C_{w}^{*}}{C_{0}}=\tau_{w}^{3}$.

In Fig. 3 there are shown relative (non-dimensional) thermal capacitances of bulk structure, ultrathin film $\left(N_{z}=3\right)$ and quantum wire $\left(N_{y}=N_{z}=3\right)$ subject to the relative temperature $\tau$. It can be seen that in low-temperature region thermal capacitance of wire is lower than that of film, and thermal capacitance of film is lower than that of massive specimens. It is also noticeable that with increase in number of layers thermal capacitance of film and wires tends toward that of bulk structure.

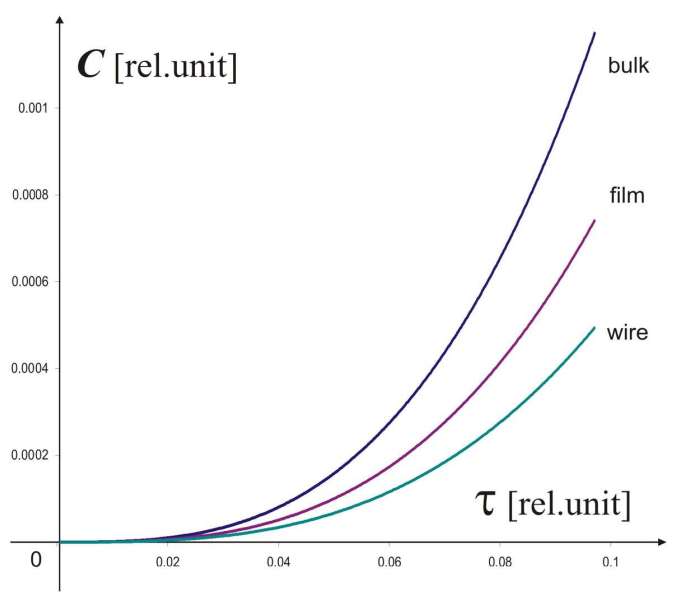

Fig. 3. Low-temperature behavior of thermal capacitance for bulk and quantum wire.

\section{Discussion}

Since phonons with the Debye frequencies are responsible for electrically and thermally induced transport properties of material [8-10], it follows that the quantum wire will be inferior electrical and thermic conductor in contrast with the relative massive structures, providing there are no chemical and structural differences between them. On the other hand, it is well known fact that the more inferior electrical conductor material is (under normal conditions), the better superconductor it becomes $[1-3$, 11-13]. Due to that, the experimental fact can be concluded and justified, that in spatially very confined structures more qualitative superconductive properties have been achieved. 
These facts point out that the key role in high $T_{\mathrm{C}}$ superconductors has the low dimension of the observed structure. The more detailed answer to this question will be obtained by examination of the electronic subsystem in quantum wires.

\section{Acknowledgments}

This work was partially supported by the Serbian Ministry of Science (grant No. 141044A) and the Ministry of Science of Republic Srpska.

\section{References}

[1] Nanostructure Physics and Fabrication, Eds. M.A. Reed, W.P. Kirk, Academic, Boston 1989, p. 49.

[2] M.G. Cottam, D.R. Tilley, Introduction to Surface and Superlattice Excitations, Univ. Press, Cambridge 1989 , p. 87.

[3] S.G. Davison, M. Steslicka, Basic Theory of Surface States, Clarendon, Oxford 1996, p. 78.

[4] J.P. Šetrajčić, V.M. Zorić, S.M. Vučenović, D.Lj. Mirjanić, V.D. Sajfert, S.K. Jaćimovski, D.I. Ilić, Mater. Sci. Forum 555, 291 (2007).

[5] S.K. Jaćimovski, J.P. Šetrajčić, B.S. Tošić, V.D. Sajfert, Mater. Sci. Forum 453-454, 33 (2004).
[6] J.P. Šetrajčić, S.K. Jaćimovski, D. Raković, D.I. Ilić, Phonon Spectra in Crystalline Nanostructures, in: Advances in Simulation, Systems Theory and Systems Engineering, Eds. N.E. Mastorakis, V.V. Kluev, Dj. Koruga, WSEAS Press, 2003, p. 146.

[7] D.I. Ilić, S.M. Vučenović, S.K. Jaćimovski, V.M. Zorić, J.P. Šetrajčić, , Proc. Materials Research Society Spring'07 Conference (San Francisco CA, USA); Symposium DD: Low-Dimensional Materials Synthesis, Assembly, Property Scaling, and Modeling, 08-50, 2007 p. 1.

[8] C. Kittel, Introduction to Solid State Physics, Wiley, New York 1986, p. 187.

[9] G. Rickayzen, Green's Functions and Condensed Matter, Academic Press, London 1980, p. 103.

[10] W. Jones, N.H. March, Theoretical Solid State Physics, Vol. 2, Dover, New York 1985, p. 87.

[11] D. Popov, S.K. Jaćimovski, B.S. Tošić, J.P. Šetrajčić, Physica A 317, 129 (2003).

[12] J.P. Šetrajčić, V.M. Zorić, S.M. Vučenović, D.Lj. Mirjanić, V.D. Sajfert, S.K. Jaćimovski, D.I. Ilić, Mater. Sci. Forum 555, 291 (2007).

[13] S.K. Jaćimovski, B.S. Tošić, J.P. Šetrajčić, V.M. Zorić, V.D. Sajfert, D.I. Ilić, Int. J. Mod. Phys. B 21, 917 (2007). 